\title{
Frequent consumption of fast foods may be associated with increased preferences for sweet and salty tastes in male university students
}

\author{
Hongmie Lee \\ Department of Food Science and Nutrition, Daejin University, Pocheon, Korea
}

\begin{abstract}
Young males are one of the most vulnerable groups to nutritional imbalance. This study attempted to determine the characteristics of male university students who frequently visit fast-food restaurants. Three hundred and forty two students attending two universities located in Daejeon and Pocheon, Korea participated for this study. Information on lifestyle, nutrition knowledge, fast-food consumption, food preferences and dietary behaviours were collected using a self-administered questionnaire. Based on the frequency of visiting fast-food restaurants, participants were categorized into three groups: rare users (less than monthly, $n$ 137), moderate users (about monthly, $n$ 98) and frequent users (more than monthly, $n$ 107). No significant differences were found among groups in age, body size, monthly pocket money, residence type and perception of own health. The frequency of visiting fast-food restaurants was not significantly associated with any of health-related lifestyle practices, such as drinking, exercise, smoking, sleeping and screen-viewing, as well as their levels of nutrition knowledge. The purposes of eating fast foods and visiting fast-food restaurants did not show significant differences between rare users and the other two groups that more often use fast food restaurants; however, a significantly less number of rare users preferred carbonated drinks $(P=0.049)$, and significantly more of them presented 'unpalatableness' as their reason to dislike fast foods $(P=0.034)$, compared to the other two groups. Also, frequent users had significantly less of desirable dietary behaviours, such as regular meals $(P=0.002)$, not skipping breakfast $(P=0.006)$, proper meal size $(P=0.032)$ and regular consumptions of protein sources $(P=0.031)$, greenish-yellow vegetables $(P=0.001)$ and vegetable oil $(P=0.004)$; and significantly more of undesirable ones such as eating out $(P=0.003)$ and frequent consumptions of processed foods $(P=0.006)$ and sweet foods $(P=0.009)$. Most of all, the results suggest that exessive intake of fast foods may result in the changes of taste preference, consequently making consumers' wanting more; frequent users had significantly higher preferences for sweet $(P=0.015)$ and salty $(P=0.031)$ tastes. Also, frequent users had significantly higher preferences for hamburgers $(P=0.000)$, fried chicken $(P=0.475)$, pizza $(P=0.034)$ and cake $(P=0.029)$, but displayed significantly lower preferences for kimchi (Korean traditional pickle) $(P=0.023)$. Although these results were based upon the information reported by subjects and did not include the actual determination of taste preferences, provide another evidence to support current dietary guidelines to reduce fast-food consumption, while awaiting confirmation in further study.
\end{abstract}

